\title{
Clinical and Histopathological Analysis of Intramucosal Zirconia Inserts used for Improving Maxillary Denture Retention
}

\author{
Fábio GONÇALVES ${ }^{1}$ \\ Eliane Pedra DIAS ${ }^{2}$ \\ Tania Mari CESTARY 3 \\ Rumio TAGA $^{3}$ \\ Raquel Virgínia ZANETTI ${ }^{1}$ \\ Artemio ZANETTI $^{1}$ \\ José Mauro GRANJEIRO ${ }^{4}$
}

${ }^{1}$ Center for the Study of Osseointegrated Implants, University City of São Paulo, São Paulo, SP, Brazil
${ }^{2}$ Department of Pathology, Medical School, Fluminense Federal University, Niteroi, RJ, Brazil
${ }^{3}$ Department of Biological Sciences, Bauru Dental School, University of São Paulo, Bauru, SP, Brazil
${ }^{4}$ Department of Cell and Molecular Biology, Institute of Biology, Fluminense Federal University, Niteroi, RJ, Brazil

Intramucosal inserts made of zirconia ceramic have been used for the rehabilitation of edentulous patients. This study aimed to follow up on patients with complete dentures having intramucosal zirconia inserts and to perform a histological analysis of biopsies of the epithelium surrounding the inserts. Twelve 31-66-year-old subjects of both genders received complete denture treatment having the intramucosal inserts in place. Detailed clinical, prosthetic and surgical procedures were described. Clinical exams were done on postoperative days $3,7,15,120$ and 360 . One year after denture placement, the subjects received local anesthesia and the tissues surrounding the insertion sites in the alveolar ridge were removed using a scalpel blade. Biopsies were fixed for up to $48 \mathrm{~h}$ in $10 \%$ phosphate-buffered formaldehyde and 5- $\mu \mathrm{m}$-thick sections were cut and stained with hematoxylin and eosin and Gomori Trichrome. Immunohistochemistry was used to identify endothelium (anti-CD3) and T lymphocytes (anti-CD31). Removing and reinserting the denture was painful until day 15, but all patients reported a marked increase in the retention and stability of the complete denture with little or no discomfort after 30 days. The histopathological analysis showed that zirconia inserts were well tolerated by the oral mucosa, with the presence of collagen fibers in the tissue around the insert, with mild inflammatory response and allowing reepithelialization, expressed by parakeratosis, epithelial hyperplasia and presenting granular layer. In conclusion, intramucosal zirconia inserts did not affect the health of oral mucosa and provide adequate retention and stability of the complete denture and comfort to the patients.

Key Words: intramucosal insert, total prosthesis, histopathology, biocompatibility, dental rehabilitation.

\section{INTRODUCTION}

The rehabilitation of edentulous patients continues to be a major challenge to dentistry. A number of techniques and materials have been investigated in order to improve retention and stability of complete dentures. The first study describing the use of intramucosal metal inserts associated with maxillary complete denture was published in the 1940s (1), in which 30 patients were followed for 10 years. During the 1950s, adjustments in the insert size and the use of non-reactive metal inserts of 2 different sizes in the same prosthesis were proposed to increase stability and retention (2). Later in the 1970s and 1980s, the focus was on the design of the retention pin with the purpose of creating retention devices, such as precision attachments (3), which would reduce the need for relining the denture and prevent dysplastic changes in the epithelium around the inserts (4). 
After the 1990s, there was a significant decrease in the number of reports on the use of intramucosal inserts, probably due to the advent of osseointegrated implants and their use in dentistry. However, a fundamental requirement for successful osseointegrated implants is the availability of sufficient quantity and quality of bone for dental implant placement (5). Bone-grafting procedures may be necessary for the reconstruction of the atrophic maxillae, increasing the complexity and morbidity of the procedure. However, oral rehabilitation using implants is not appropriate for a large number of patients due to restrictions, such as local or systemic conditions that contraindicate implant placement, high-cost procedures and patient refusal.

Recently, intramucosal inserts made of zirconia $\left(\mathrm{ZrO}_{2}\right.$ or zirconium oxide) ceramic have become available (6). This ceramic material has high strength $(7)$, is inert and biocompatible $(8,9)$ and has been used as abutments (10). The zirconia inserts have a highly polished surface that minimizes the formation and accumulation of biofilms (11). In spite of the well-known biocompatibility of zirconia, no clinical or histological studies have been performed to evaluate the effect of the use of intramucosal inserts made of zirconia ceramic on the epithelium in contact with such devices.

This purpose of this study was to follow up on patients with complete dentures having intramucosal zirconia inserts, and to perform a histological analysis of biopsies of the epithelium surrounding the inserts.

\section{MATERIAL AND METHODS}

\section{Zirconia Intramucosal Inserts}

Very well polished intramucosal inserts (Muchor ${ }^{\circledR}$; Dyna Dental Engineering B.V., PW Bergen Op Zoom, The Netherlands) made of zirconium oxide $\left(\mathrm{ZrO}_{2}, \mathrm{Y}_{2} \mathrm{O}_{3}\right.$ yttrium stabilized) were used in this study. Figure 1 shows the 2-part insert: the elliptical part of the insert (3.2 $\mathrm{mm}$ diameter and $5.2 \mathrm{~mm}$ height) remains in contact with the mucosa and the other part is retained into the acrylic denture base ( $4.4 \mathrm{~mm}$ diameter).

\section{Study Sample}

Twelve subjects of both genders aged 31 to 66 years received complete denture treatment in the Center for the Study of Osseointegrated Implants at the Uni- versity City of São Paulo (UNICID/CEIO). This study followed the principles of the Declaration of Helsinki and the informed consent was obtained from all patients after a clear and complete explanation of the study purposes had been provided.

In the present study, eligibility criteria included patients who were experiencing problems due to lack of retention of complete dentures, alveolar bone loss and maxillary atrophy with loose mucosa and pneumatized maxillary sinuses. Patients were excluded if they had chronic systemic disorders, such as uncontrolled diabetes and hypertension, were not interested in the procedure and refused to cooperate, or did not fulfill the clinical and radiographic criteria required for the placement of osseointegrated implants. Eligibility and exclusion criteria were verified by clinical interview, clinical examinations and panoramic radiograph. Table 1 summarizes the characteristics of the subjects selected for the study.

Initially, the subjects underwent surgical procedures to prepare the mouth to receive the complete denture, with the removal of anatomical or pathological elements that could contribute to the lack of stability of the maxillary complete denture. Hyperplastic mucosa was removed by cryosurgery followed by the use of tissue conditioners to promote tissue repair.

In the construction of complete dentures, maxillary and mandibular dental impressions were taken, casts were prepared and mounted in the articulator using the Zanetti technique (12), intermaxillary registration was

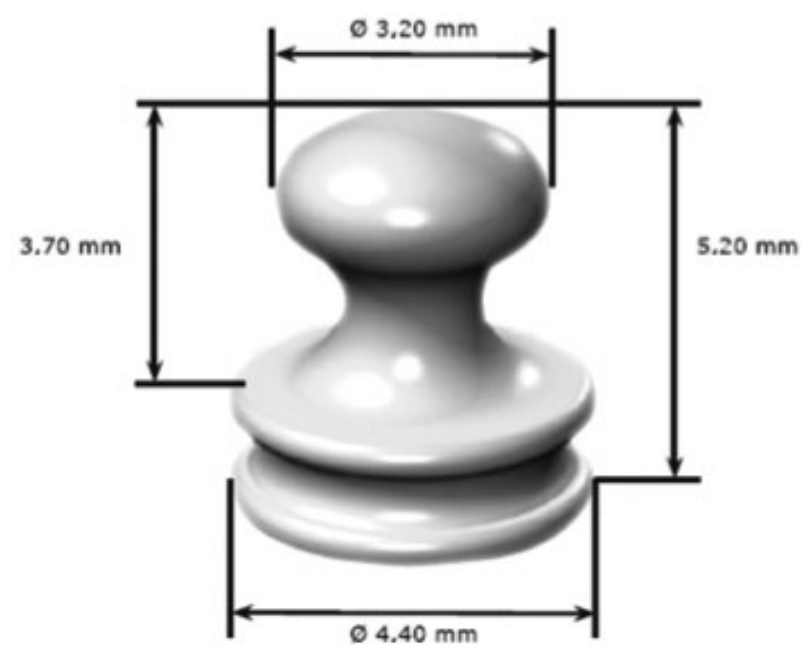

Figure 1. Photograph showing the shape and dimensions of zirconia intramucosal inserts. 
performed, the denture teeth best suited for each patient were selected and the dentures were inserted. Adjustments for overextension and relieve of pressure areas, as well as occlusal adjustments were made during the first 15 days following denture insertion.

After being adapted and balanced, each complete denture was relined with zinc oxide-eugenol cement and sent to the dental laboratory. Also, an acrylic guide plate was made to mark the positions for the denture inserts.

A dental stone cast was made from the relined denture and an adequate number of intramucosal inserts required to meet the needs of each patient were placed on it using a surveyor to ensure a proper insertion path and facilitate the further construction of the denture. An acrylic surgical guide was made from the stone cast having the intramucosal inserts in place in order to assure a uniform and correct positioning of the preparation sites in the mucosa. The denture was then invested and, after acrylic resin polymerization, it was removed from the mold and polished.

With the surgical guide in place, the injection sites of local anesthesia were identified. The anesthesia was strictly limited to the locations for the insert preparation sites in the mucosa, thus avoiding excess of anesthesia or anesthesia of the posterior vestibule, which could result

Table 1. Characteristics of the subjects using intramucosal inserts made of zirconia ceramic and postoperative follow-up visits at which biopsies of the mucosa surrounding the insertion sites were performed.

\begin{tabular}{cccc}
\hline Case & Gender & Age (years) & $\begin{array}{c}\text { Follow-up visit } \\
\text { (months) }\end{array}$ \\
\hline 1 & Female & 60 & 12 \\
2 & Male & 54 & 12 \\
3 & Male & 58 & 12 \\
4 & Female & 62 & 12 \\
5 & Female & 31 & 12 \\
6 & Female & 55 & 36 \\
7 & Male & 57 & 12 \\
8 & Female & 66 & 18 \\
9 & Male & 55 & 15 \\
10 & Female & 37 & 18 \\
11 & Male & 55 & 12 \\
12 & Female & 49 & 12 \\
\hline
\end{tabular}

${ }^{1}$ All study subjects were Caucasians. ${ }^{2}$ All retention devices were inserted in the mucosa covering the maxillary alveolar ridge. in local or peripheral edema that would interfere with the full insertion of the denture. Five-millimeter-deep preparation sites were made using a special drill at low speed under water cooling supplied by the manufacturer of the inserts. After the preparation sites were made and adjusted, the denture was inserted and checked for fitness, assuring full insertion. A small amount of dental adhesive paste was applied to the palatal area of the denture to increase stability and initial retention, contributing to tissue repair of the mucosa around the intramucosal inserts. All patients were instructed not to remove the denture for $72 \mathrm{~h}$, the time taken for the initial healing of the mucosa around the inserts. After the 3rd day, the patients could remove the denture only for cleaning and then return it to the mouth.

\section{Clinical Evaluation}

The subjects returned to the study center (CEIOUNICID) on postoperative days 3, 7, 15, 120 and 360. At these follow-up visits, the clinical aspect of the mucosa in contact with the insert was subjectively evaluated, as well as denture retention and stability, patient comfort when eating, removing and reinserting the denture, and speech alterations.

\section{Biopsy and Histopathological Evaluation}

After a minimal period of 1 year following denture insertion, the subjects received local anesthesia and the tissues surrounding the insert sites in the alveolar ridge were removed with a \#10 scalpel blade. All biopsies were fixed for up to $48 \mathrm{~h}$ in $10 \%$ phosphate-buffered formaldehyde ( $\mathrm{pH}$ 7.2) and sent to the Laboratory of Pathology of the Antônio Pedro University Hospital, Fluminense Federal University, RJ, Brazil. After macroscopic analysis, the biopsies were dehydrated in increasing ethanol concentrations, cleared in xylol, and immersed in liquid paraffin at $60^{\circ} \mathrm{C}$. After obtaining the paraffin blocks, 5 - $\mu$ m-thick sections were cut and stained with hematoxylin and eosin and Gomori Trichrome. Histological evaluation was conducted by an experienced pathologist. The endothelium and $\mathrm{T}$ lymphocytes were identified by immunohistochemistry using anti-CD3 and anti-CD31 antibodies, respectively, as described before (13).

Soon after, $6-\mu \mathrm{m}$-thick sections were cut from the paraffin-embedded tissues and were mounted on 
silane-coated glass slides (Super Frost Plus; Erviegas, São Paulo, SP, Brazil). The immunohistochemical characterization of cells was performed using the avidinbiotin-peroxidase complex (ABC) method. Slides were deparaffinized in xylol and ethanol, and incubated in $3 \%$ hydrogen peroxide diluted in phosphate-buffered saline (PBS) (pH 7.2) for $40 \mathrm{~min}$ at room temperature. The slides were then immersed in citrate buffer $(\mathrm{pH}$ 6.0) (P4809; Sigma Chemical Co., Saint Louis, MO, USA) for $20 \mathrm{~min}$ at $950^{\circ} \mathrm{C}$ for antigen retrieval. Soon after, the slides were incubated in 3\% normal rabbit serum diluted 1:100 in distilled water for $20 \mathrm{~min}$ at room temperature, and were sequentially incubated with rabbit anti-human $\mathrm{CD} 3$ and $\mathrm{CD} 31$ polyclonal antibodies diluted 1:100 in 1\% PBS-bovine serum albumin (BSA) overnight at $40^{\circ} \mathrm{C}$.

Following the incubation period, the slides were washed with PBS and incubated with biotinylated goat anti-rabbit IgG antibody diluted 1:100 in 1\% PBS-BSA for $60 \mathrm{~min}$ at room temperature. Then, the slides were incubated in avidin-biotin complex (K0492; Dako, Carpinteria, CA, USA) for $45 \mathrm{~min}$ at room temperature, followed by incubation in a solution of $5 \mathrm{mg}$ of 3,3-diaminobenzidine (D4293; Sigma) diluted in $10 \mathrm{~mL}$ of PBS containing 180 $\mu \mathrm{L}$ of $20 \%$ hydrogen peroxide for 20 to $30 \mathrm{~min}$ at room temperature. After washing with distilled water, the slides were counterstained with Harris hematoxylin for $5 \mathrm{~min}$. Tissue samples from giant cell tumors of bone were used as positive controls. Negative controls were obtained by substituting the primary antibodies with $1 \%$ PBS-BSA and normal rabbit serum (X0903; Dako).

\section{RESULTS}

\section{Clinical Evaluation}

On postoperative day 3 , the patients reported pain when removing and reinserting the denture, which had been held in place by dental adhesive paste. A yellowish cicatricial tissue was present at the insertion sites between postoperative days 3 and 7 . On postoperative day 15 , patients were still on a pureed diet and showed some improvement in the retention of the denture, but still reported pain on its removal and reinsertion. On postoperative day 30 , all patients reported a marked increase in the retention and stability of the maxillary complete denture with no need for dental adhesive, were able to eat a normal diet, and felt little or no discomfort when removing and reinserting the denture. The appearance of the mucosa at the insertion sites was identical to the palatal mucosa. During this time, no patient developed any local infectious process related to the insertion sites.

At quarterly follow-up visits, there was little need for occlusal adjustment or adjustment of denture borders. On postoperative day 360 , only 1 subject required the removal of 2 inserts from the denture to reduce its retention due to difficulties in removing the prosthesis. Aspect of normal oral mucosa was found after 3 years (Fig. 2).

\section{Histopathological Evaluation}

The macroscopic examination revealed mucosa fragments varying in size from $0.4 \times 0.4 \times 0.2$ to $0.8 \times$

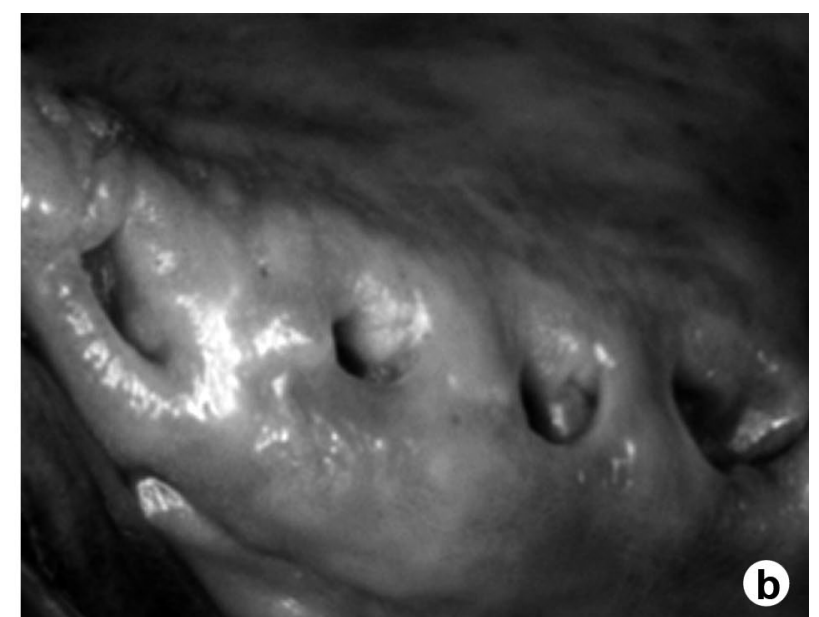

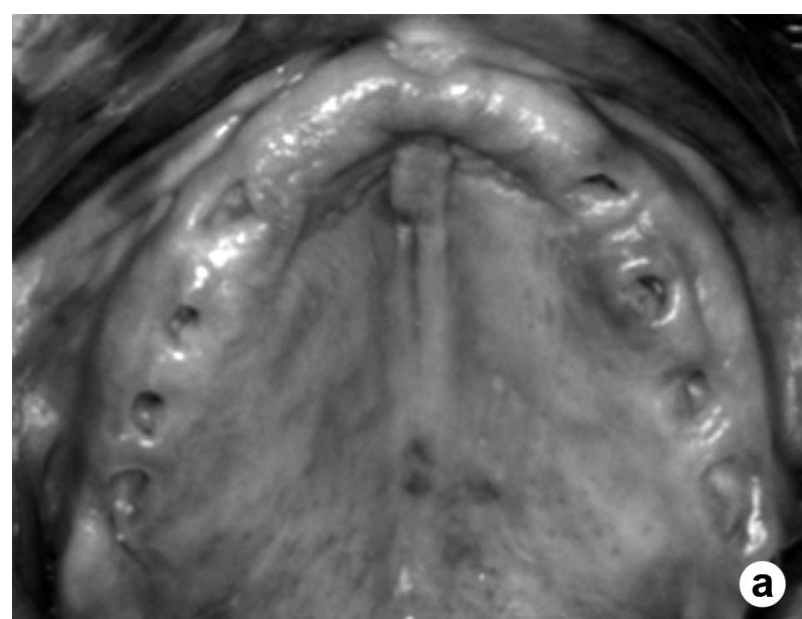

Figure 2. Clinical aspect of the maxillary alveolar ridge 3 years after surgery showing normal oral mucosa around the insertion sites (A, B). 
$0.7 \times 0.4 \mathrm{~cm}$, with an average volume of $0.08 \mathrm{~cm}^{3}$, brown color, elastic, and showing central hole lined with white mucosa. The microscopic analysis (Figs. 3A and B) revealed 2 patterns in the analyzed slides: (a) oral mucosa of the surgical margin: mild keratinization, moderate epithelial hyperplasia, keratinocytes with perinuclear halos, moderate spongiose, and mononuclear exocytosis; and (b) periorificial oral mucosa (in contact with the insert): parakeratosis, mild to severe epithelial hyperplasia, minimal to extensive granular layer; lamina propria with dense collagen bands (Fig. 3C), fading of the loose subepithelial connective tissue, few mononuclear leukocytes (scar). The focal mononuclear infiltrate, which was composed predominantly of $\mathrm{T}$ lymphocytes, was confirmed by anti-CD3 (Fig. 3D). Sparse new formed vessels, well evidenced by anti-CD31 (Figs. 3E and F).

\section{DISCUSSION}

The histopathological observations performed in this study indicate that polished zirconia ceramic is very well tolerated by the mucosal tissue, leading to the accumulation of collagen fibers in the area around the insert, maintaining mild inflammatory response, and allowing reepithelialization, which is expressed by parakeratosis, epithelial hyperplasia and presenting granular layer. No granulomatous reaction or important inflammatory foci was observed. Microscopic observations suggest that zirconia ceramic can be an effective alternative material for use in intramucosal inserts. This is in agreement with the very good results obtained from clinical evaluations, which showed that the use of inserts leads to a marked increase in the retention of the denture, thus providing comfort and confidence to patients. The data reported here are in strong accordance with those of previous studies $(14,15)$.

This technique was suggested by Hans Nordgren and developed by Gustav
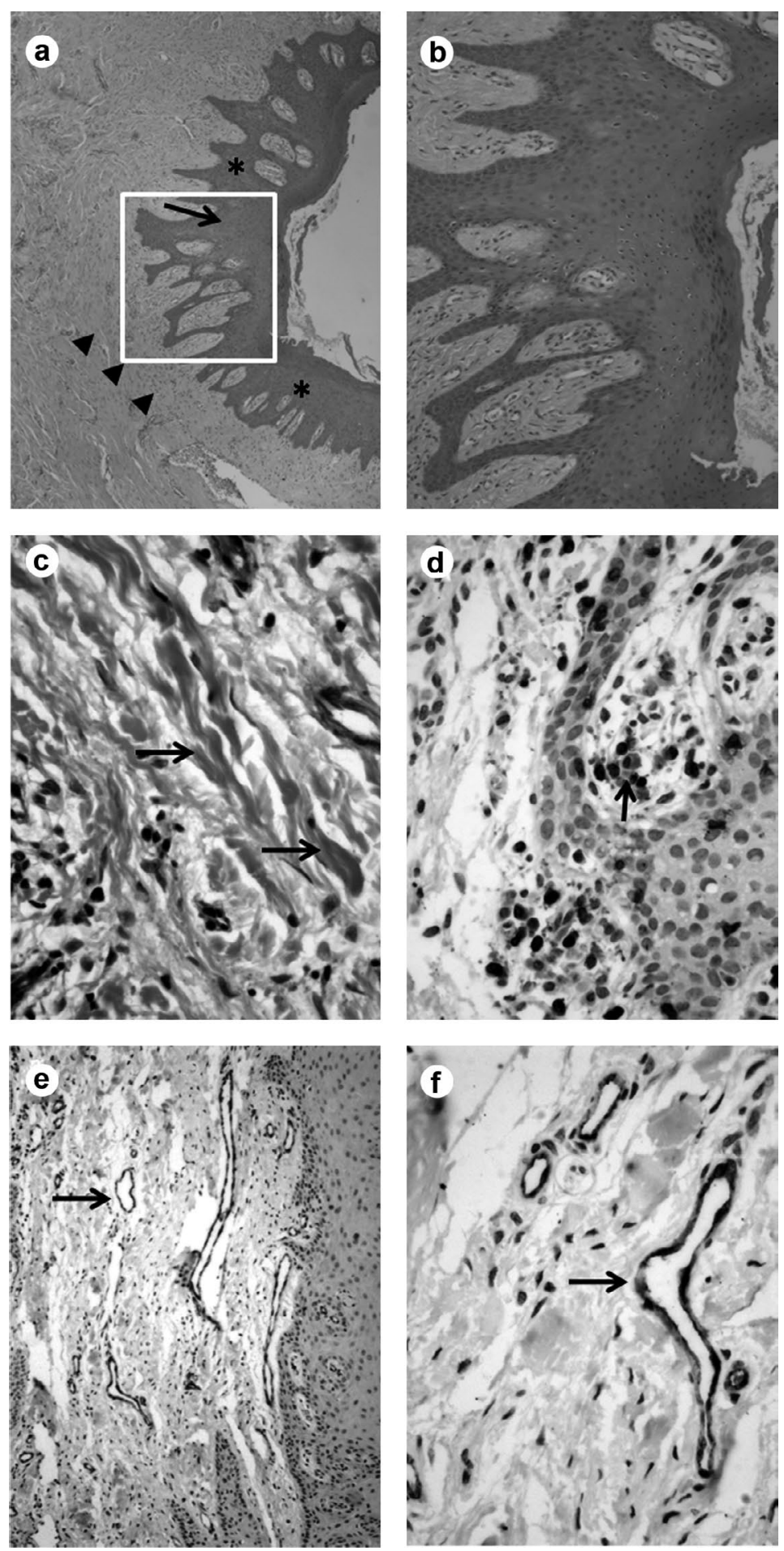

Figure 3. Histopathological features. A. Periorificial oral mucosa with parakeratosis $(*)$, granular layer, epithelial hyperplasia (arrow) and fibrosis (arrow head, HE). B. Detail: granular layer (arrows), parakeratosis, and mild subepithelial inflammatory infiltrate (HE). C. Fibrosis (arrows, Gomori Trichrome). D. Immunohistochemistry: T lymphocytes stained with anti-CD3. E-F Immunohistochemistry: endothelial cells stained with anti-CD31 (arrow). Original magnifications: A. $20 \times$, B. $100 \times$, C-E. $400 \times$, F. $100 \times$. 
Dahl (1) at the end of the first half of the 20th century. It makes use of intramucosal metal inserts, which according to the histopathological analysis, promotes the formation of a densely fibrous connective tissue around the insert, showing sclerosed vessels, metabolically active basal layer and parakeratosis (16). After some time, the insertion site in the palatal mucosa may become hyperkeratinized or show histological evidence of mild chronic inflammation. However, to date there are no reports of patient complaints. Clinically, the insertion site was healthy without edema or other signs of inflammation, showing pink mucosa of normal appearance. Dahl (1), a pioneer in this area, reported the use of mushroomshaped stainless steel or chrome-cobalt alloy inserts with sizes proportional to the thickness of the mucosa, thereby facilitating their insertion and improving denture retention. Another important aspect of this technique is to place the insertion sites slightly to the palatal side of the alveolar ridge, where the submucosa is thicker.

Along the years, the most important modifications to this technique have been focused on the shape of the insert, which had always been made of metal alloys. The present article reports for the first time the use of highly polished zirconia ceramic as the insert. It was observed that the replacement of metal by zirconia did not produce noticeable changes in the cellular response to the inserts. Previous work has shown the safety, biocompatibility and stability of zirconia ceramics (17-19), and the lack of mutagenic and carcinogenic effect (20).

The laboratory and surgical procedures are very simple and ease to perform by the general clinician. The observed clinical responses reflect the healing process of the oral mucosa after surgical procedures; the healing process was completed within 30 postoperative days. The cooperation of the patient is extremely important during this stage for the patient must keep the denture in place as long as possible, even during periods of discomfort.

The clinical results obtained for the treated patients confirmed a marked increase in the retention of the complete maxillary denture in all cases. All 12 patients reported improvement in their diets due to an increased ability to chew semisolid and solid foods. As a consequence, the patients' self-esteem and confidence increased, thus improving the quality of their social interactions. However, it is important to point that the development of the technique to obtain the described results never neglected the principles of the construction of a maxillary complete denture. In other words, although the use of intramucosal inserts increase the retention of complete dentures, it is important not to overlook the principles of occlusal balance and stability, neither stop the search for methods to achieve better casting accuracy in recreating anatomical features. It should be noted that that we are still dealing with a complete denture and precise measurements of the vertical dimension and intermaxillary relations are also required for complete dentures with intramucosal inserts.

Based on both clinical and histopathological observations it can be concluded that intramucosal zirconia inserts did not affect the mucosal health and provide adequate retention and stability of the complete denture and comfort to the patients. Patients did not report any complaints after wearing the denture with zirconia inserts for more than 1 year, and no alterations were observed in the insertion sites that could impact the mucosal health.

\section{REFERENCES}

1. Dahl GS. Some aspects of the use of intramucosal inserts. J Oral Implant Transplant Surg 1966;12:61-65.

2. Cranin AN, Cranin SI. The intramucosal insert: a method of maxillary denture stabilization. J Am Dent Assoc 1958;57:188-193.

3. Guaccio RA. Intramucosal inserts for retention of removable maxillary prostheses. Dent Clin North Am 1980;24:585-592.

4. Misch CE. An improved design and technique for mucosal inserts. J Oral Implantol 1982;10:41-54.

5. Schimming R, Schmelzeisen R. Tissue-engineered bone for maxillary sinus augmentation. J Oral Maxillofac Surg 2004;62:724-729.

6. Hisbergues M, Vendeville S, Vendeville P. Zirconia: Established facts and perspectives for a biomaterial in dental implantology. $\mathrm{J}$ Biomed Mater Res B Appl Biomater 2009;88:519-529.

7. Raigrodski AJ. Contemporary materials and technologies for allceramic fixed partial dentures: a review of the literature. J Prosthet Dent 2004;92:557-562.

8. Scarano A, Di CF, Quaranta M, Piattelli A. Bone response to zirconia ceramic implants: an experimental study in rabbits. J Oral Implantol 2003;29:8-12.

9. Takamori ER, Cruz R, Gonçalvez F, Zanetti RV, Zanetti A, Granjeiro JM. Effect of roughness of zirconia and titanium on fibroblast adhesion. Artif Organs 2008;32:305-309.

10. Welander M, Abrahamsson I, Berglundh T. The mucosal barrier at implant abutments of different materials. Clin Oral Implants Res 2008;19:635-641.

11. Scotti R, Kantorski KZ, Monaco C, Valandro LF, Ciocca L, Bottino MA. SEM evaluation of in situ early bacterial colonization on a Y-TZP ceramic: a pilot study. Int J Prosthodont 2007;20:419-422.

12. Zanetti AL, Ribas R. A new method to simplify and increase the precision of maxillary cast mounting procedures in fully adjustable or semiadjustable articulators. J Prosthet Dent 1997;77:219-224.

13. Accorsi-Mendonca T, Zambuzzi WF, da Silva Paiva KB, Pereira L Jr., Cestari TM, Taga R, et al.. Expression of metalloproteinase 2 in the cell response to porous demineralized bovine bone matrix. J Mol Histol 2005;36:311-316. 
14. Massimei GG. Template construction for accurate placement of intra-mucosal inserts. Implantologist 1978;1:103-111.

15. Evasic RW. Intramucosal implants. A review of concepts and techniques. Single inserts and tandem denserts. J Oral Implantol 1982;10:9-23.

16. Cranin AN, Cranin SI. The intramucosal insert: a method of maxillary denture stabilization. J Am Dent Assoc 1958;57:188-193.

17. Ichikawa Y, Akagawa Y, Nikai H, Tsuru H. Tissue compatibility and stability of a new zirconia ceramic in vivo. J Prosthet Dent 1992;68:322-326.
18. Takamura K, Hayashi K, Ishinishi N, Yamada T, Sugioka Y. Evaluation of carcinogenicity and chronic toxicity associated with orthopedic implants in mice. J Biomed Mater Res 1994;28:583-589.

19. Cales B. Zirconia as a sliding material: histologic, laboratory, and clinical data. Clin Orthop Relat Res 2000;379:94-112.

20. Covacci V, Bruzzese N, Maccauro G, Andreassi C, Ricci GA, Piconi $\mathrm{C}$, et al.. In vitro evaluation of the mutagenic and carcinogenic power of high purity zirconia ceramic. Biomaterials 1999;20:371376.

Acepted April 8, 2009 